\title{
Oscillatory Behavior of Solutions of Fourth-order Mixed Neutral Difference Equations with Asynchronous Non Linearities
}

\author{
Shirmila Premkumari. K
}

\begin{abstract}
In this article, oscillation criteria for solutions of fourth order mixed type neutral difference equation with asynchronous non linearities of the form
\end{abstract}

$\Delta^{2}\left(a_{n} \Delta^{2}\left(x_{n}+b_{n} x_{n}-\tau_{1}+c_{n} x_{n+\tau_{2}}\right)\right)+q_{n} x^{a_{n}+1-\sigma_{1}}+p_{n} x_{n+1+\sigma_{2}}^{\beta}=0$

where $\{a n\},\{b n\},\{c n\},\{q n\}$ and $\{p n\}$ are established. Examples are provided to illustrate the results.

Keywords: Oscillation, Neutral difference equation, asynchronous.

\section{INTRODUCTION}

Neutral difference equations exist in stability theory, exist theory, network systems and so on. It has applications in problems dealing with vibrating masses to elastic bar and variational problems. Consider the fourth-order mixed type neutral difference equation with asynchronous non linearities of the form

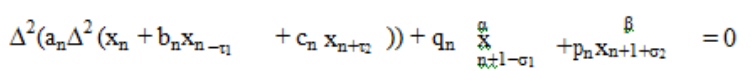

where $\left\{a_{n}\right\},\left\{b_{n}\right\},\left\{c_{n}\right\},\left\{p_{n}\right\}$ and $\left\{q_{n}\right\}$ are positive real sequences, $\alpha$ and $\beta$ are ratios of positive odd integers $\tau_{1}, \tau_{2}$, $\sigma_{1}$ and $\sigma_{2}$ are positive integers and $n \in N$ where $N=\left\{n_{0}, n_{0}\right.$ $\left.+1, \mathrm{n}_{0}+2, \ldots\right\}, \mathrm{n}_{0}$ is a non negative integers. The forward difference operator is defined by $\Delta x_{n}=x_{n+1}-x_{n}$.

Let $\theta=\max \left\{\tau, \sigma_{1}\right\}$. By a solution of (1) a real sequence $\left\{x_{n}\right\}$ which is defined for all $\mathrm{n} \geq \mathrm{n}_{0}-\theta$ and satisfies equation (1) for all $n \in N$. A non trivial solution $\left\{x_{n}\right\}$ is said to be non oscillatory if it is either eventually positive or eventually negative and it is oscillatory otherwise .

In the past two years there has been an increasing interest in the study of oscillatory behavior of solution of difference equations. See [1-10] and reference cited therein. If $\alpha=\beta$ in (1) then it is a synchronous case. If $\alpha=\beta$, then (1) is an equation with asynchronous non linearities.

In this paper we discuss the oscillatory and asymptotic behavior of solutions of equations with asynchronous non linearities.

\section{SOME PRELIMINARY LEMMAS}

In this section, we present some oscillation criteria for equation (1). For all suf- ficiently large $n$, consider a functional inequality holds and assume the following

Revised Manuscript Received on丸uly 08, 2019.

ShirmilaPremkumari. K, Karunya Institute of Technology and Sciences, Coimbatore, India.abiramidarshan74@gmail.com conditions.

(H1) $\left\{a_{n}\right\}$ is a positive non-decreasing sequence such that $\sum_{n=n_{0}}^{\infty} \frac{1}{a_{n}}=\infty$;

$(H 2)\left\{b_{n}\right\}$ and $\left\{c_{n}\right\}$ are real sequences such that $0 \leq b_{n}$ $\leq \mathrm{b}$ and $0 \leq \mathrm{c}_{\mathrm{n}} \leq \mathrm{c}$ with

$$
\mathrm{b}+\mathrm{c}<1 \text {; }
$$

(H3) $\left\{P_{n}\right\}$ and $\left\{Q_{n}\right\}$ are positive real sequences;

(H4) $\alpha$ and $\beta$ are both rations of odd positive integer $\tau_{1}$, $\tau_{2}, \sigma_{1}$ and $\sigma_{2}$ are nonnegative integers.

Result:

Here we adopt the following notations:

$\mathrm{y}_{\mathrm{n}}=\mathrm{x}_{\mathrm{n}}+\mathrm{b}_{\mathrm{n}} \mathrm{x}_{\mathrm{n}}-\tau 1+\mathrm{c}_{\mathrm{n}} \mathrm{x}_{\mathrm{n}}+\tau_{2}$

$\mathrm{Q}_{\mathrm{n}}=\min \left\{\mathrm{q}_{\mathrm{n}}, \mathrm{q}_{\mathrm{n}}-\tau 1, \mathrm{q}_{\mathrm{n}+\tau 2}\right\}$

$P_{n}=\min \left\{P_{n_{\beta}} P_{n}-\tau 1, P_{n}+\tau 2\right\}$

$\mathrm{W}_{\mathrm{S}}=\mathrm{Q}_{\mathrm{S}}+\mathrm{M}^{\beta-\alpha} \mathrm{P}_{\mathrm{S}}$

Lemma 2.1. Assume $A \geq 0, B \geq 0, \alpha \geq 1(A+B){ }^{\alpha} \leq 2^{\alpha-1}\left(A^{\alpha}\right.$ $\left.+\beta^{\alpha}\right)($ see $[11])$.

Lemma 2.2.Let $\left\{x_{n}\right\}$ be a positive solution of equation (1). Then there are two cases hold for $n \geq n_{1} \in N$ sufficiently large:

(1) $\mathrm{y}_{\mathrm{n}}>0, \Delta \mathrm{y}_{\mathrm{n}}>0, \Delta^{2} \mathrm{y}_{\mathrm{n}}>0, \Delta\left(\mathrm{a}_{\mathrm{n}} \Delta^{2} \mathrm{y}_{\mathrm{n}}\right) \leq 0, \Delta^{2}\left(\mathrm{a}_{\mathrm{n}} \Delta^{2} \mathrm{y}_{\mathrm{n}}\right) \leq$ 0 ;

(2) $y_{n}>0, \Delta y_{n}<0, \Delta^{2} y_{n}>0, \Delta\left(a_{n} \Delta^{2} y_{n}\right) \leq 0, \Delta^{2}\left(a_{n} \Delta^{2} y_{n}\right) \leq$ 0 .

(see [11])

Lemma 2.3. Let $\mathrm{y}_{\mathrm{n}}>0, \Delta \mathrm{y}_{\mathrm{n}}>0, \Delta^{2} \mathrm{y}_{\mathrm{n}}>0, \Delta^{3} \mathrm{y}_{\mathrm{n}} \leq 0$, $\Delta^{4} \mathrm{yn}_{\mathrm{n}} \leq 0$ for all

$\mathrm{n} \geq \mathrm{N} \in \mathrm{N}$. Then for any $\xi \in(0,1)$ and some integer $\mathrm{N}_{1}$, the following inequalities

$$
\frac{\mathrm{y}_{\mathrm{n}+1}}{\Delta \mathrm{y}_{\mathrm{n}}} \geq \frac{\mathrm{n}-\mathrm{N}}{2} \geq \frac{\xi_{\mathrm{n}}}{2}
$$

for $\mathrm{n} \geq \mathrm{N}_{1}>\mathrm{N}$ hold.

Lemma 2.4. Suppose that $\left\{x_{n}\right\}$ be a positive solution of equation (1) with the upper bound $\mathrm{M}$, and the corresponding yn satisfies (2) of lemma 2.2. Also if $\sum_{n=n_{0}}^{\infty} \sum_{g=n}^{\infty}\left(\frac{1}{a_{s}} \sum_{t=s}^{\infty}\left(q_{t}+M^{\beta-\infty} P_{t}\right)\right)=$ $\infty$

holds, then $\lim n \rightarrow \infty \mathrm{x}_{\mathrm{n}}=0$.

Proof.

Let $\left\{x_{n}\right\}$ be a positive solution of equation (1) satisfying $x_{n}$ $\leq \mathrm{M}$.

$\lim \mathrm{yn}_{\mathrm{n}}=\lambda 0$ exists. It can be proved that $\lambda=0$. If not, $\lambda>0$, 
and for any

$\mathrm{n} \rightarrow \infty$

$>0$, we have $\lambda+\epsilon>$ eventually. Choose

$0<\epsilon<\frac{\lambda(1-b-c)}{b+c}$

then we have $\quad \mathrm{x}_{\mathrm{n}}=\mathrm{y}_{\mathrm{n}}-\mathrm{b}_{\mathrm{n}} \mathrm{x}_{\mathrm{n}}-\tau 1-\mathrm{c}_{\mathrm{n}} \mathrm{x}_{\mathrm{n}}+\tau_{2}$

$>\lambda-(\mathrm{b}+\mathrm{c}) \mathrm{yn}_{\mathrm{n}}-\tau 1$

$>\lambda-(b+c)(\lambda+E)$

$=\mathrm{g}(\lambda+\epsilon)$

$>$ gyn

where

$\mathrm{g}=>0$.

$$
\lambda-(b+c)(\lambda+E)
$$

$\lambda+\epsilon$

Further,

$$
\begin{aligned}
\Delta\left(\mathrm{a}_{\mathrm{n}} \Delta \mathrm{y}_{\mathrm{n}}\right) \leq & -\mathrm{q}_{\mathrm{n}} \mathrm{g}^{\alpha} \mathrm{y}^{\alpha} \quad{ }_{n+1-\sigma_{1}}-P_{n} g x_{\mathrm{n}+1-\sigma_{2}}^{\beta, \beta} \\
& \leq-\mathrm{g}^{\alpha}\left(\mathrm{q}_{\mathrm{n}}+\mathrm{M}^{\beta-\alpha} \mathrm{P}_{\mathrm{n}}\right) \mathrm{y}_{n+1-T_{1}}^{\alpha}
\end{aligned}
$$

Summing the above inequality from $\mathrm{n}$ to $\infty$, and using the relation $y_{n} \geq \lambda$, we have

$\Delta^{2} y_{n} \geq(g \lambda)^{\alpha}\left(\frac{1}{a_{n}} \sum_{\gamma=n}^{\infty}\left(q_{Y}+M^{\beta-\alpha_{p}} p_{\gamma}\right)\right)$

Summing the two sides of (4) from $n$ to $\infty$

$-\Delta y_{n} \geq(g \lambda)^{\alpha}\left(\sum_{g=n}^{\infty} \frac{1}{\omega_{s}} \sum_{t=s}^{\infty}\left(q_{t}+M^{\beta-\alpha_{p}} p_{t}\right)\right)$

Since, $\mathrm{y}_{\mathrm{n}}>0, \Delta \mathrm{y}_{\mathrm{n}}<0$.

Summing from $\mathrm{n}_{1}$ to $\infty$ leads to

$y_{n_{1}} \geq(g \lambda)^{\alpha} \sum_{n=n_{1}}^{\infty} \sum_{s=n}^{\infty} \frac{1}{a_{s}} \sum_{t=s}^{\infty}\left(q_{t}+M^{\beta-a_{p}} p_{t}\right)$

which is a contradiction to (3). Then $\lambda=0$, which together with the inequality

$0<x_{n}<y_{n}$ implies that $\lim x_{n}=0$. The proof is complete.$$
\mathrm{n}
$$$$
\rightarrow
$$$$
\infty
$$

Let $\mathrm{Q}_{\mathrm{n}}=\min \left\{\mathrm{q}_{\mathrm{n}}, \mathrm{q}_{\mathrm{n}}-\tau 1, \mathrm{q}_{\mathrm{n}+\tau 2}\right\}, \mathrm{P}_{\mathrm{n}}=\min \left\{\mathrm{P}_{\mathrm{n}}\right.$, $\left.\mathrm{P}_{\mathrm{n}-\tau 1}, \mathrm{P}_{\mathrm{n}+\tau 2}\right\}$

Theorem 2.1. Suppose that $\left\{x_{n}\right\}$ be a bounded positive solution of equation (1) with the upper bound $M$, and the condition (3) holds, $\sigma_{1} \geq \tau_{1}$ and $\alpha_{1} \geq \beta \geq 1$. If there exists a positive real sequence $\left\{\eta_{n}\right\}$ and an integer $N_{1} \in$ such that for some $\xi \in(0,1)$ and $\delta>0$.

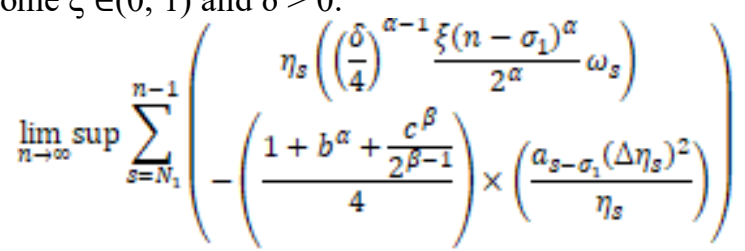

holds, then every such solution $\left\{x_{n}\right\}$ of equation (1) oscillates or $\lim _{n \rightarrow \infty} x_{n}=0$.

\section{Proof.}

Let $\left\{x_{n}\right\}$ be a non oscillatory solution of equation (1), and $x_{n}$ $\leq \mathrm{M}$. Let that there exists an integer $\mathrm{N} \geq \mathrm{n}_{0}$ such that $\mathrm{x}_{\mathrm{n}}$, $x_{n}-\sigma 1, x_{n}+\sigma_{2}, x_{n}-\tau 1, x_{n}+\tau_{2} \in(0, M]$ for all $n>N$, we have,

$$
\begin{aligned}
& \Delta^{2}\left(a_{n} \Delta^{2} y_{n}\right)+a_{n} x^{a}+a+1-\sigma_{1}+P_{n} x_{n+1+\sigma 2}^{\beta}+b^{a} \Delta^{2}\left(a_{n}-q \Delta^{2} y_{n}-n\right)
\end{aligned}
$$

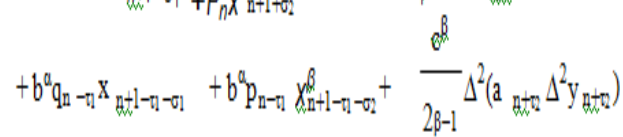

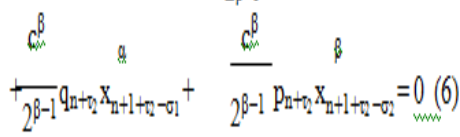

By lemma (2.1) and $\beta \leq \alpha$ in (6), we have

$$
\begin{aligned}
& \Delta^{2}\left(a_{n} \Delta^{2} y_{n}\right)+b^{\alpha} \Delta^{2}\left(a_{n-\tau_{1}} \Delta^{2} y_{n-\tau_{1}}\right) \\
& d Q_{n} M^{\beta-\alpha} \\
& 2^{\beta-1} 4^{\alpha-1 n+1-\sigma_{1} 4^{\alpha-1 n+1+\sigma_{2}}}+-\Delta^{2}\left(a_{n+\tau_{2}} \Delta^{2} y_{n+\tau_{2}}\right)+y^{\alpha}+y^{\alpha}
\end{aligned}
$$

By lemma 2.2, there are two cases for $y_{n}$ to be considered. Assume that (1) holds for all $n \geq N_{1} \geq N$.

$$
\begin{aligned}
& \text { It follows from } \Delta y_{n}>0, \text { that } y_{n}+\sigma 2>y_{n}-\sigma 1 \\
& \Delta^{2}\left(a_{n} \Delta^{2} y_{n}\right)+b^{\alpha} \Delta^{2}\left(a_{n}-r_{1} \Delta^{2} y_{n}-\tau 1\right) \\
& c^{\beta}
\end{aligned}
$$

$+\Delta^{2}\left(\operatorname{an}+\tau_{2} \Delta^{2} y n+\tau_{2}\right)+Q_{n}$
$2^{\beta-1} y^{\alpha-1 n+1-\sigma 1}$

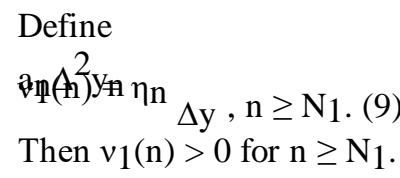

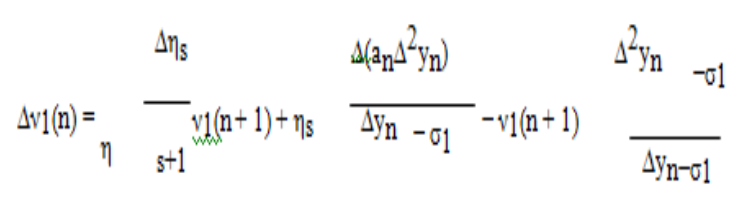

$$
\begin{aligned}
& \Delta^{2}\left(a_{n} \Delta^{2} Z_{n}\right)=-q_{n} x^{a}-p_{n} x<<0(10) \\
& n+1-\sigma_{n} n+1+\sigma_{21} \\
& \text { and } 22
\end{aligned}
$$

$$
a_{n-\sigma !} \Delta y_{n-\sigma]} \geq a_{n+1} \Delta y_{n+1}
$$

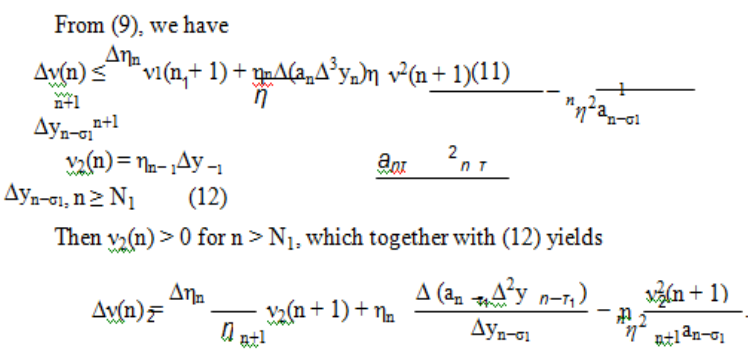

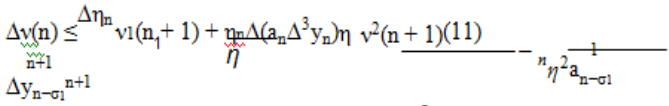

Similarly define

$$
v_{3}(n)=\eta_{n} \frac{a_{n t \tau_{2}} \Delta y^{2}{ }_{n+\tau_{2}}}{\Delta y_{n-\sigma_{1}}}, n \geq N_{d i}
$$

Then we have,

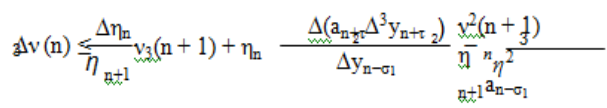

Published By: 


$$
\begin{aligned}
& \Delta v_{1}(n)+b^{a} \Delta v_{2}(n)+\frac{c^{b}}{2^{\beta-1}} \Delta v_{3}(n)
\end{aligned}
$$

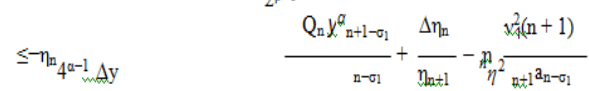

$$
\begin{aligned}
& +b^{a} \times \frac{\Delta \eta_{n} v_{2}(n+1)}{\eta_{n+1}}-\eta_{n_{1}} \frac{v_{2}^{2}(n+1)}{\beta_{2}^{2} a_{n}-\sigma_{1}}
\end{aligned}
$$

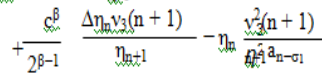

Since $\left\{a_{n}\right\}$ is nondecreasing and $\Delta^{2} y_{n}>0, \Delta^{3} y_{n}<0, \Delta^{4} y_{n}<0$ for $n \geq N_{1}$. By lemma (2.3) for any

$$
\frac{\mathrm{y}_{\mathrm{n}+1-\sigma_{1}}}{\Delta \mathrm{y}_{\mathrm{n}-\sigma_{1}}} \geq \frac{\stackrel{\xi}{K}\left(\mathrm{n}-\sigma_{1}\right)}{2}
$$

$c^{\beta}$

$\Delta v_{2}(n)+b^{a} \Delta v_{2}(n)+\Delta v_{3}(n) \leq \overline{-\eta_{n}}{ }^{a-1} \xi\left(n-\sigma_{1}\right)^{a} Q_{3}+M^{\beta-4} P_{n}$

$2^{\beta-14} \quad 2^{a}$

$1+b^{a}+\underline{d}_{a_{n-11}}\left(\Delta \eta_{m}\right)^{2}$

$+$

Summing the inequality from $\mathrm{N}_{2} \geq \mathrm{N}_{1}$ to $\mathrm{n}-1$.

$$
\begin{gathered}
\sum_{s=N_{2}} \eta_{s}\left(\left(\frac{\delta}{4}\right)^{\alpha-1} \frac{\xi\left(n-\sigma_{1}\right)^{\alpha}}{2^{\alpha}}\left[Q_{s}+M^{\beta-\alpha} P_{s}\right]\right)+\frac{(1}{} \\
\leq v_{1}\left(N_{2}\right)+b^{\alpha} v_{2}\left(N_{2}\right)+\frac{c^{\beta}}{2^{\beta-1}} v_{a}\left(N_{2}\right)
\end{gathered}
$$

Let $\eta_{\mathrm{n}}=\mathrm{N}, \alpha=\beta=1$. Then the following corollary is easily obtained.

Corollary 2.1. Suppose that $\left\{x_{n}\right\}$ be a bounded positive solution of equation (1) with the upper bound $M$, and $\alpha=1$. Assume that (5) holds and $\sigma_{1}>\tau_{1}$. If there is an integer $\mathrm{N}_{1}$ $\in \mathrm{N}$ such that for some $\xi \in(0,1)$ and $\delta>0$.

$\left.\lim _{n \rightarrow \infty} \sup _{s=N_{1}} \sum_{\eta_{s}}\left(\frac{\delta}{4}\right)^{\alpha-1} \frac{\xi\left(n-\sigma_{1}\right)^{n} \alpha}{2^{\alpha}} W_{s}-\frac{1+b+c}{4 s} a_{s-\sigma_{1}}\right)$

holds, then every such solution $\left\{x_{n}\right\}$ of (1) oscillate (or) $\lim _{n \rightarrow \infty} x_{n}=0$, where

$$
\mathrm{W}_{\mathrm{S}}=\mathrm{Q}_{\mathrm{S}}+\mathrm{M}^{\beta-\alpha} \mathrm{P}_{\mathrm{S}} \text {. }
$$

Theorem 2.2. Suppose that $\left\{x_{n}\right\}$ be a bounded positive solution of equation (1) with the upper bound $M$. Assume that the condition (7) holds, $\sigma_{1}<\mathrm{T}_{1}$, and $\alpha \geq \beta \geq 1$. If there exists a positive real sequence $\eta_{\mathrm{n}}$, and an integer $\mathrm{N}_{1} \in$ such that for some $\xi \in(0,1)$ and $\delta>0$

$\lim _{n \rightarrow \infty} \sup _{s=N} \sum_{s}^{n-1}\left(\frac{\delta}{4}\right)^{\alpha-1} \frac{\xi\left(n-\sigma_{1}\right)^{n} \alpha}{2^{s}} \omega_{s}-\frac{\left(1-b^{\alpha}+\frac{c^{\beta}}{2^{\beta}-1}\right) a_{s-\tau_{s}}}{4 \tau_{s}}$

holds, then every solution $\left\{x_{n}\right\}$ of equation (1) oscillates or

$$
\lim _{n \rightarrow \infty} x_{n}=\text {. }
$$

\section{Proof.}

Assume that case 1 of lemma 2.2 holds for all $n \geq N_{1} \geq \mathbb{N}$. Then define,

$v_{1}(n)=\eta_{n} \frac{a_{n} \Delta^{2} y_{n}}{\Delta y_{n-\tau_{1}}}, \quad n \geq N_{1}$,

$v_{2}(n)=\eta_{n} \frac{a_{n-\tau_{1}} \Delta^{2} y_{n-\tau_{1}}}{\Delta y_{n-\tau_{1}}}, \quad n \geq N_{1}$.

$v_{a}(n)=\eta_{n} \frac{a_{n+\tau_{2}} \Delta^{2} y_{n+\tau_{2}}}{\Delta y_{n+\tau_{2}}}, \quad n \geq N_{1}$.

We have,

$$
\begin{gathered}
\Delta v_{1}(n)+b^{\alpha} v_{2}(n)+\frac{c^{\beta}}{2^{\beta-1}} \Delta v_{a}(n) \leq-\eta_{n} \frac{Q_{n}}{4^{\alpha-1}} \frac{Z_{n+1-\tau_{1}}^{\infty}}{\Delta y_{n-\tau_{1}}}+\frac{\Delta \eta_{n}}{\eta_{n+1}} \\
-\eta_{n} \frac{\omega_{1}^{2}(n+1)}{\eta_{n+1}^{2} a_{n-\tau_{1}}}+b^{\alpha}\left(\frac{\Delta \eta_{n} v_{2}(n+1)}{\eta_{n+1}}-\eta_{n} \frac{\omega}{\eta_{n}}\right. \\
+\frac{c^{\beta}}{2^{\beta-1}}\left(\frac{\Delta \eta_{n} v_{3}(n+1)}{\eta_{n+1}}-\eta_{n} \frac{\omega_{2}^{2}(n+1)}{\eta_{n+1}^{2} a_{n-\tau_{1}}}\right)
\end{gathered}
$$

On the other hand, we have for any $\xi \in(0,1)$

$\frac{y_{n+1-\sigma_{1}}}{\Delta y_{n-\tau_{1}}}=\frac{y_{n+1-\sigma_{1}}}{\Delta y_{n-\sigma_{1}}} \frac{y_{n+1-\sigma_{1}}}{\Delta y_{n-\tau_{1}}} \geq \frac{\xi\left(n-\sigma_{1}\right)}{2}$

for all $n \geq N_{2}$, we obtain

$$
\Delta v_{1}(n)+b^{\alpha} v_{2}(n)+\frac{c^{\beta}}{2^{\beta-1}} \Delta v_{a}(n) \leq-\eta_{n} \omega_{n}+\frac{\left(1+b^{\alpha}+\frac{c^{\beta}}{2^{\beta-1}}\right) a_{n}}{4 \gamma_{n}}
$$

The proof is similar to theorem 2.1 Summing the inequality from $\mathrm{N}_{2}$ to $\mathrm{n}-1$, we obtain

$$
\sum_{s=\mathbb{N}_{2}}^{n-1} \eta_{s}\left(\left(\frac{\delta}{4}\right)^{\alpha-1} \frac{\xi\left(n-\sigma_{1}\right)^{\alpha}}{2^{\alpha}}\left[Q_{s}+M^{\beta-\alpha} P_{s}\right]\right)+\left(1+b^{\alpha}+\frac{c^{\beta}}{2^{\beta-1}}\right) a_{s}
$$


$\sigma_{1}=3, \sigma_{2}=0, \alpha=2 \leq \beta=3$.

Take $\eta_{\mathrm{n}}=1$. It is easy to verity that (3) hold. On the otherhand, condition (5) is also true.

Therefore by theorem (6), every Solution $\left\{x_{n}\right\}$ of $(25)$

$\lim _{n \rightarrow \infty} x_{n}=0$

Example 3.2. Consider the fourth order difference equation

$\Delta^{2}\left(a_{n} \Delta^{2}\left(x_{n}+b_{n} x_{n-\tau_{1}}+c_{n} x_{n+\tau_{2}}\right)\right)+\frac{c}{n} x_{n+1-\sigma_{1}}^{2}+\frac{d}{n} x_{n+1+\sigma_{2}}=0$

wherec, $d$ are positive constant, $0 \leq \mathrm{b}_{\mathrm{n}} \leq \mathrm{b}, 0 \leq \mathrm{c}_{\mathrm{n}} \leq \mathrm{c}$ and $\mathrm{b}$ $+\mathrm{c}<1$. Let

$\mathrm{a}_{\mathrm{n}}=\mathrm{n} \mathrm{b}_{\mathrm{n}}=c_{\mathrm{n}}=\frac{1}{\mathrm{a}}, \mathrm{q}_{\mathrm{n}}=\frac{\mathrm{c}}{\mathrm{n}}, \mathrm{p}_{\mathrm{n}}=\frac{\mathrm{d}}{\mathrm{n}}, \mathrm{\sigma}=2>\beta=1$. Take $\eta_{\mathrm{n}}$. It is easy to see that theorem (6), (or) theorem (8) is well satisfied.

Therefore every solution $\left\{x_{n}\right\}$ of equation (26) oscillates (or)

$\lim _{n \rightarrow \infty} x_{n}=$.

\section{CONCLUSION}

In this paper we solve non linear differential equation and inequality of differential equations in our real life problems. We find new theorem, definition and lemmas of those inequalities and those non linear differential equations.

\section{REFERENCES}

1. R.P.Agarwal, S.R.Grace, and J.Graef. Oscillation Criteria for certain Third Order Nonlinear Difference Equations. Appl. Anal. Discrete Math., 3:27-38, 2009

2. M.Bohner, R.P.Agarwal, and D.O'Regan. Discrete Oscillation Theory. New York:HindawiPubl Co, 2005.

3. S.Donthaand, S.R.Grace. Oscillation of Higher Order Neutral Difference Equations of Mixed Typed. Dynam.Systems. J. Math. Anal. Appl., 12:521$532,2003$.

4. S.R.Grace. Oscillation of certain Neutral Difference Equations of Mixed Typed. J. Math. Anal. Appl., 224:241-254, 1988.

5. S.R.Grace and R.P.Agarwal. The Oscillation of certain Difference Equations. Math.Comput.Modelling, 30:53-66, 1999.

6. S.R.Grace and R.P.Agarwal. Oscillation of Higher Order Nonlinear Difference Equations of Neutral type. Appl. Math. Lett., 12:77-83, 1999.

7. S.R.Grace andR.P.Agarwal. Oscillation of certain Third-Order Difference Equations.Comput.Math. Appl., 42:379-384, 2001.

8. S.R.Grace, R.P.Agarwal, and E.A.Bohner. On the Oscillation of Higher Order Neutral Difference Equations of Mixed type.Dynam. Systems Appl., 11:459- 470, 2002.

9. S.R.Grace, R.P.Agarwal, and P.J.Y.Wong. On the Oscillation of Third Order Nonlinear Difference Equations. J. Appl. Math. Comput., 32:189-203, 2010.

10. N.Kavitha and E.Thandapani. Oscillatory Behavior of Solutions of certain Third Order Mixed Neutral Difference Equations. Acta.Math. Sci., 33B(1) : 218-226, 2013

\section{AUTHORS PROFILE}

ShirmilaPremkumari. K, Karunya Institute of Technology and Sciences, Coimbatore, India.abiramidarshan74@gmail.com 